\title{
Geoportal de suelos de Costa Rica como Bien Público al servicio del país
} Geoportal of Costa Rican soils as Public
Good at the service of the country

Rafael Mata-Chinchilla ${ }^{1}$, Jonathan Castro-Chinchilla²

Mata-Chinchilla, R; Castro-Chinchilla. Geoportal de suelos de Costa Rica como Bien Público al servicio del país. Tecnología en Marcha. Vol. 32, Especial. XIII CLIA. Abril 2019. Pág 51-56.

DOI: https://doi.org/10.18845/tm.v32i7.4259

1 Máster en Ciencias del Suelo, docente e investigador del Centro de Investigaciones Agronómicas, Universidad de Costa Rica. Costa Rica. Correo electrónico: rafael. mata@ucr.ac.cr

2 Máster en Gestión de la Innovación tecnológica, Especialista en Proyectos del Instituto Interamericano de Cooperación para la Agricultura, IICA. Cost Rica. Correo electrónico: jonathan.castro@iica.int. 


\title{
Palabras clave
}

Suelos; Costa Rica; geoportal; SIG.

\section{Resumen}

Poco más de 364.000 datos sobre características físicas, químicas y taxonómicas de suelos, de 1565 puntos de muestreos georreferenciados se encuentran ahora accesibles mediante la plataforma virtual "Geoportal de Suelos de Costa Rica", en la dirección web www.suelos.cr.

Este es un esfuerzo liderado por el Centro de Investigaciones Agronómicas de la Universidad de Costa Rica, junto a el Instituto Nacional de Innovación y Transferencia en Tecnología Agropecuaria (INTA), la Asociación Costarricense de la Ciencia del Suelo (ACCS) y el Instituto Interamericano de Cooperación para la Agricultura (IICA).

Si bien la cartografía digital de suelos se empezó en Costa Rica en la década de 1990, sucesivos avances tecnológicos y actualizaciones en la taxonomía edáfica han obligado a abordar de manera innovadora la organización de vastísima información disponible, pero dispersa.

El trabajo desarrollado ha requerido el diseño, gestión y aseguramiento de la calidad de dos productos principales, una base de datos homogénea y la representación geoespacial mediante Sistemas de Información Geográfica (SIG). Productos intermedios del proceso se han materializado como Protocolos para la Gestión de Información en la Base de Datos, una sistematización metodológica del proceso, así como soportes digitales y materiales multimedia (videos) para la utilización de los productos digitales.

Los próximos pasos para la plataforma Geoportal de Suelos de Costa Rica incluyen su difusión y el monitoreo del aprovechamiento por los usuarios, el incremento de la densidad y oferta de datos manteniendo su acceso libre y la posibilidad de exportar el modelo de gestión a otros países del hemisferio.

\section{Keywords}

Soils; Costa Rica; Geoportal; GIS.

\begin{abstract}
Just over 364,000 data on physical, chemical and taxonomic soil characteristics, of 1565 georeferenced sampling points are now accessible through the virtual platform "Geoportal de Suelos de Costa Rica", at the web address www.suelos.cr.

This is an effort led by the Center for Centro de Investigaciones Agronómicas of the Universidad de Costa Rica, together with the Instituto Nacional de Innovación y Transferencia en Tecnología Agropecuaria (INTA), la Asociación Costarricense de la Ciencia del Suelo (ACCS) and the InterAmerican Institute of Cooperation for Agriculture (IICA).

Although digital soil cartography was started in Costa Rica in the 1990s, successive technological advances and updates in the edaphic taxonomy have forced us to address in an innovative way the organization of a vast amount of information available but dispersed.

The work developed has required the design, management and assurance of the quality of two main products, a homogeneous database and geospatial representation through Geographic Information Systems (GIS). Intermediate products of the process have materialized as Protocols
\end{abstract}


for the Management of Information in the Database, a methodological systematization of the process, as well as digital media and multimedia materials (videos) for the use of digital products.

The next steps for the Geoportal Platform of Soils of Costa Rica include its dissemination and monitoring of the use by users, the increase in the density and supply of data while maintaining its free access and the possibility of exporting the management model to other countries of the hemisphere.

\section{Introducción}

El Autor Klaus Schwab propone que actualmente estamos confrontados al desarrollo de la Cuarta Revolución Industrial. Esta Cuarta Revolución básicamente implica un fuerte cambio desde la actual aplicación de tecnologías digitales a la resolución de problemas y la técnica (Tercera Revolución Industrial), hacia una inmersión en las tecnologías digitales, como parte del diario vivir y las innovaciones disruptivas en íntimo y estrecho vínculo con las actividades humanas. Tres aspectos son fundamentales en los cambios actuales de paradigma, la velocidad de las transformaciones del entorno, la amplitud y profundidad con que intervienen en la sociedad y las personas y, por último, su impacto en los sistemas, donde está reorganizando la forma de hacer las cosas [1].

La posición de Costa Rica respecto a esta Cuarta Revolución Industrial, es relativamente modesta. En el informe 2017 del Índice Global de Innovación, el país se ubica en el puesto 53 de 127 estados evaluados, cayendo 8 lugares respecto a su ubicación en el 2016 [2]. Los riesgos de este rezago respecto a la innovación y tecnología ya habían sido advertidos para el Primer Informe del Estado de la Ciencia y la Tecnológica, ECTI, Costa Rica, y en particular para el ámbito agropecuario nacional. El Estado de la Nación determinó en el año 2014 que la mayoría de los grupos científicos en el país se caracterizan por una alta "centralidad", en la que prevalece un escaso número de investigadores, la mayoría de los cuales se encuentra en edad madura (el 71,9\% tiene 46 años o más). Se observan debilidades en el campo de las Ciencias Agrarias, debido a un limitado capital de relaciones entre sus miembros [3].

En el ámbito de las ciencias del suelo históricamente se ha trabajado en la generación de productos científicos y técnicos de cobertura nacional, en diversos formatos. Por ejemplo, en el año de 1979 el Ministerio de Agricultura y Ganadería publica en formato análogo el Mapa de Suelos de Costa Rica escala 1:200.000 [4]. Este mismo autor cambia de formato este producto, llevándolo a su primera versión digital en el año 1989.

A partir del 2012 un grupo conformado por representantes de la Universidad de Costa Rica, Asociación Costarricense de las Ciencias del Suelo (ACCS) y del Instituto Nacional de Innovación y Transferencia en Tecnología Agropecuario (INTA), inician el proceso para revisar, corregir y digitalizar los mapas de órdenes de suelos existentes a la fecha. Paralelamente a este proceso se inicia con la compilación y revisión de perfiles de suelos realizados desde 1975 hasta el 2012 que permanecían en papel y conformar una base digital de datos de perfiles que sustentara las correcciones y edición del mapa de órdenes y subórdenes de suelos. El 5 de diciembre del 2013, se celebró el día Mundial de Suelos y en el marco de esta celebración se publicó el nuevo mapa de órdenes y subórdenes de suelos de Costa Rica a una escala 1: 200.000 y la base de datos con 450 perfiles de suelos de Costa Rica [5]. A este esfuerzo se suma en 2015 el apoyo del Instituto Interamericano de Cooperación para la Agricultura, IICA, en su Representación Costa Rica, para una nueva actualización que sale a la luz en el año 2016. Esta actualización incluye una ampliación de la base de datos alcanzando poco más de 1500 perfiles incluidos y su desarrollo bajo el enfoque de un Bien Público al servicio de la agricultura costarricense [6]. 
Si bien el producto presentado implica un esfuerzo de muchos años y entidades, así como una herramienta de alto potencial para el análisis nacional desde el enfoque edáfico hacia la producción agropecuaria, su gestión aun requería un nivel de especialización medio alto por parte del usuario. Al ser presentado como insumos de un Sistema de Información Geográfica, requiere el conocimiento y uso, por ejemplo, de programas informáticos especializados y conocimientos relacionados. Es por esto, que un nuevo esfuerzo coordinado entre el CIA-UCR y el IICA para el año 2017 buscó presentar tanto el Mapa de Digital Suelos de Costa Rica versión 2016, como su base de datos, a través de un formato digital en línea, ampliando su acceso a múltiples usuarios, no necesariamente especializados.

\section{Materiales y métodos}

El desarrollo del geo portal de suelos de Costa Rica se basa en los insumos obtenidos en la actualización del Mapa Digital de Suelos de Costa Rica versión 2016, incluidos una base de datos construida con el programa Microsoft Excel® y una capa digital en formato Sistema de Información Geográfica.

La base de datos fue tanto diseñada como alimentada por personal asociado al CIA-UCR. Los datos que le dieron origen incluyen una amplia serie de publicaciones de dominio público, incluidos artículos científicos, libros, tesis, entre otros. El requisito fundamental para su inclusión en la base de datos se definió por la disponibilidad de una descripción detallada del suelo asociada a un sitio georreferenciado concreto.

La base de datos de suelos complementada y actualizada fue sujeta de sucesivas revisiones y controles de calidad, que incluían uniformidad de la referencia geográfica, la nomenclatura y coherencia de la profundidad de horizontes, uniformidad de unidades en que se expresan características físicas y químicas, entre otras. Con los resultados depurados se realizaron enmiendas y actualizaciones, especialmente en términos de taxonomía de suelos (Orden, Suborden, Gran grupo, Sub grupo). De ser necesario y según la representatividad de los datos, de las enmiendas a la base de datos se realizaron correcciones y mejoras a las capas digitales del Mapa de Suelos.

Tomando como base el producto digital en línea GoogleMaps (), se superpusieron las capas digitales de Órdenes y Subórdenes de suelos, utilizando base de programación informática HTML. Asimismo, los puntos de referencia geográfica para cada sitio incluido y descrito en la base de datos. Se incluyó un recuadro de navegación, que describe la ubicación del puntero sobre el mapa en términos de coordenadas geográficas y el correspondiente Orden y Suborden de suelo. Un nombre de dominio especifico fue registrado en NIC Costa Rica.

El Geo portal de Suelos fue asociado a una cuenta de correo independiente, como buzón de intercambio con el usuario (opción "Contáctenos"). Esta misma dirección de correo fue asociada a la utilidad GoogleAnalitics (C), con la finalidad de dar seguimiento a los accesos al Geo Portal, sitios visitados y set de datos de puntos de muestreo descargados. Lo anterior con la finalidad de determinar las tendencias de consulta y utilización de la herramienta digital.

\section{Resultados}

La totalidad de información compilada originó una base de datos que reúne información de 1565 puntos de muestreo a lo largo y ancho de Costa Rica. De estos sitios de muestreo se capturan poco más de 364.000 datos sobre características físicas, químicas y taxonómicas de suelos. Como máximo 93 variables de caracterización de los suelos incluyen (cuando la fuente original de datos lo contenía): 
- Número de identificación del punto (ld)

- Longitud y latitud (WGS 84)

- Ubicación Política (Provincia, Cantón, Distrito)

- Año de descripción

- Número, nombre y profundidad de horizontes

- Para cada horizonte: pH en agua, Acidez, Ca, Mg, K, Suma de bases, CICE (como $\mathrm{cmol}(+) / L)$, Saturación de acidez (como \%), P, Zn, Cu, Fe, Mn (como mg/L), Materia y Carbono Orgánico (como \%), Bases en Acetato de Amonio (como cmol(+)/kg), ClC, Saturación de Bases (como \%), pH en NaF, pH en KCl, Retención P (como \%), Al y Fe en oxalato (como \%), Conductividad Eléctrica $(\mathrm{mS} / \mathrm{cm})$, Arena, Limo y Arcilla (como \%), Clase Textural, Granulometría de la fracción gruesa (como gr/cm3), Densidad Aparente y de Partículas (como \%), Poros (como \%), Retención de humedad y agua útil (como \%), Conductividad hidráulica (como cm/h), Resistencia a la penetración (como N/cm2), COLE, Provincia fisiográfica, Material Parental, Clima Edáfico, Zona de Vida, Relieve, Gradiente (como \%), Altitud (en m.s.n.m.), Dirección de la pendiente, Orientación, Vegetación, Fertilidad Aparente, Drenaje, Profundidad de la tabla de agua, Pedregosidad/Roca Superficial, Erosión, Color de la Matriz, Color acompañante, Estructura, Consistencia, Porosidad, Raíces, Limites, Biomasa Microbiana, Formaciones especiales,

- Clasificación taxonómica USDA (Orden, Suborden, Gran grupo, Sub grupo) y Autor del estudio

Las capas digitales presentadas en el geoportal describen 1091 polígonos que cubren la totalidad del área continental de Costa Rica, en los niveles de Órdenes y Subórdenes de suelos. Presentan un total de 21 categorías de Órdenes o transiciones y 39 categorías de Subórdenes o transiciones.

Los 1565 puntos de muestreo se despliegan para su revisión preliminar presentando los datos: Provincia, Cantón, Distrito, Latitud, Longitud, Orden, Suborden, Gran Grupo, Subgrupo, Cantidad de horizontes descritos, Nombre de los Horizontes descritos y Total de datos químicos y físicos disponibles para el punto. La descarga de todas las variables descritas en la base de datos, como formato Microsoft Exel (C) está disponible para los usuarios, sin requerimientos de registro o costo. La plataforma se puso a disposición en la dirección web http://suelos.cr/ (figura 1).

\section{Conclusiones}

El Geoportal de suelos puede considerarse el primer esfuerzo unificado en dotar de total accesibilidad y sin costo a datos relevantes sobre los suelos de Costa Rica, tanto a público especializado como a la sociedad civil en general. Este instrumento trata de alinear el conocimiento generado desde hace muchos años y por muchos actores a los modos actuales de gestión de datos bajo formatos digitales y plataformas abiertas de Sistemas de Información Geográfica.

Este debe considerarse como un primer esfuerzo, ampliamente mejorable, donde se espera, a partir de la experiencia del usuario (estadísticas de uso y realimentación directa), avanzar en dos líneas importantes, 1) incrementar la disponibilidad de datos y 2) mejorar la forma de presentarlos para su utilización en aplicaciones específicas. 


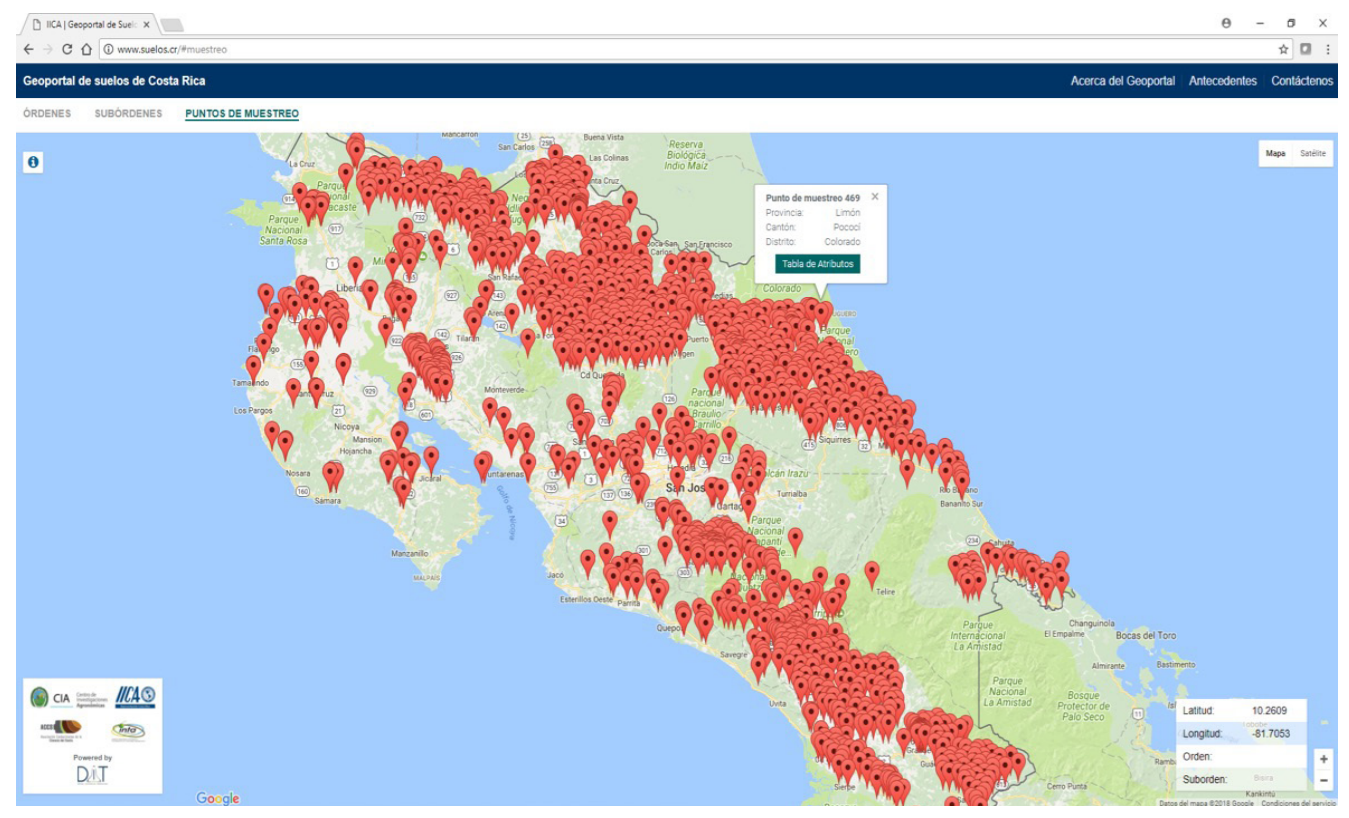

Figura 1. Visualización del Geoportal de Suelos de Costa Rica 2017.

Si bien el producto parte de un robusto proceso técnico y de un innovador estilo de acceso y presentación, procura también establecer un nuevo paradigma de colaboración. Este parte de las asociaciones iniciales para lograr el avance actual (CIA-UCR, ACCS, INTA e IICA), pasa por las diferentes entidades y personas que han aportado datos y propone una continuidad de un modelo colaborativo para ampliar el esquema. Esto incluye tanto la sistematización como el acceso al conocimiento de suelo, como punto de partida parar una amplísima gama de productos que pueden derivarse en mejora del sector primeramente agropecuario, y posiblemente en otros ámbitos relacionados a la disciplina de los suelos.

\section{Referencias}

[1] K. Schwab, La cuarta revolución industrial. Madrid, España: Editorial Debate, 2016, pp 224.

[2] S. Dutta, B. Lanvin, y S. Wunsch-Vincent, The global innovation index 2017: Innovation feeding the world. Johnson Cornell University, 2017.

[3] P. E. de la Nación, «Estado de la ciencia, la tecnología y la innovación», San José, Costa Rica, 2014, pp. 405.

[4] A. Vásquez, «Mapa de suelos de Costa Rica escala 1: 200.000», Ministerios de Agricultura y Ganadería. San José, Costa Rica, 1979.

[5] CIA-UCR, «Mapa digital de Suelos de Costa Rica». San José de Costa Rica. 2013.

[6] IICA. «Costa Rica actualiza su mapa digital de suelos | Instituto Interamericano de Cooperación para la Agricultura». [En línea]. Disponible en: http://www.iica.int/es/prensa/noticias/costa-rica-actualiza-su-mapa-digital-de-suelos. 